\title{
Avaliação de conceitos de termodinâmica clássica através de mapas conceituais
}

Guilherme Cordeiro da Graça de Oliveira

Paula Macedo Lessa dos Santos

Rodrigo dos Santos Almeida

\section{Resumo}

Neste trabalho o mapeamento conceitual foi utilizado para avaliação de conceitos de termodinâmica clássica, numa turma do curso de Engenharia Química da Universidade Federal do Rio de Janeiro, como parte da primeira avaliação da disciplina. Foi proposto aos alunos que construíssem um mapa conceitual com 8 conceitos obrigatórios e, no máximo, 4 conceitos complementares. A metodologia empregada na avaliação dos mapas elaborados pelos alunos consistiu na contagem e análise das proposições. Os principais objetivos foram: (i) investigar a aplicação dos mapas conceituais como instrumento de avaliação dos conteúdos de termodinâmica clássica; (ii) avaliar as proposições construídas pelos alunos e (iii) investigar a percepção dos alunos com relação à metodologia de avaliação utilizando mapas conceituais. Os resultados permitiram concluir que os alunos apresentaram uma boa compreensão dos conceitos investigados, porém identificaram também concepções errôneas em desacordo com a visão científica. Entrevistas semiestruturadas revelaram uma boa aceitação da metodologia de avaliação através de mapas conceituais. química.

Palavras-chave: mapas conceituais, termodinâmica clássica, ensino de

\section{Abstract}

\section{Evaluation of classical thermodynamic concepts through concept maps}

In this paper the conceptual mapping was used to evaluate concepts of classical thermodynamics in a class of Chemistry Engineering of Federal University of Rio de Janeiro. It was proposed to students to make a conceptual map involving 8 compulsory concepts and, at most, 4 complementary concepts. The methodology employed to evaluate the conceptual maps consisted in the counting and analysis of propositions constructed by students with compulsory and complementary concepts. The main objectives were: (i) investigate the application of maps as a tool for evaluating of classical thermodynamics content; (ii) evaluate the propositions constructed and (iii) to investigate the students' perceptions regarding the assessment methodology using concept maps. The results showed that students had a good understanding of the investigated concepts, but also misconceptions identified at odds with the scientific view. Semistructured interviews showed a good acceptance of the evaluation methodology using concept maps.

Keywords: conceptual maps, classical thermodynamics, chemistry 
teaching.

\section{Introdução}

Os mapas conceituais (MC) foram desenvolvidos em 1972 por um grupo liderado por Joseph D. Novak num programa de pesquisa da Universidade de Cornell (Estados Unidos) diante da necessidade de se interpretar como as crianças compreendiam os conceitos científicos (Novak e Cañas, 2006). Este programa se baseava na psicologia da aprendizagem de David Ausubel, a aprendizagem significativa. A partir de então surgiu uma ferramenta poderosa capaz de organizar e representar o conhecimento - não só em relação às crianças - e que pode ser utilizada tanto em pesquisa educacional quanto em vários outros domínios do conhecimento.

As unidades cognitivas fundamentais dos $\mathrm{MC}$ são as proposições, as quais são formadas por dois conceitos ligados por um termo de enlace. Novak e Cañas $(2006$, p. 1) definiram conceito como "uma regularidade percebida em eventos ou objetos, designada por um rótulo que, por sua vez, pode ser constituído por um símbolo (tal como + ou \%), por uma ou mais palavras". Os termos de enlace são as palavras ou frases que ligam os conceitos formando uma unidade semântica lógica. A Figura 1 apresenta o exemplo de um mapa conceitual simples descrevendo uma colmeia (Cline, Brewster e Fell, 2010, p. 2283). Os conceitos encontram-se dentro de retângulos e os termos de enlace são "contem" e "produz". As setas indicam o sentido de leitura das proposições que devem assumir clareza semântica. Por exemplo, para o conceito "zangão" aparecem no mapa duas proposições que o relaciona com os conceitos "colmeia" e "pupa de zangão".

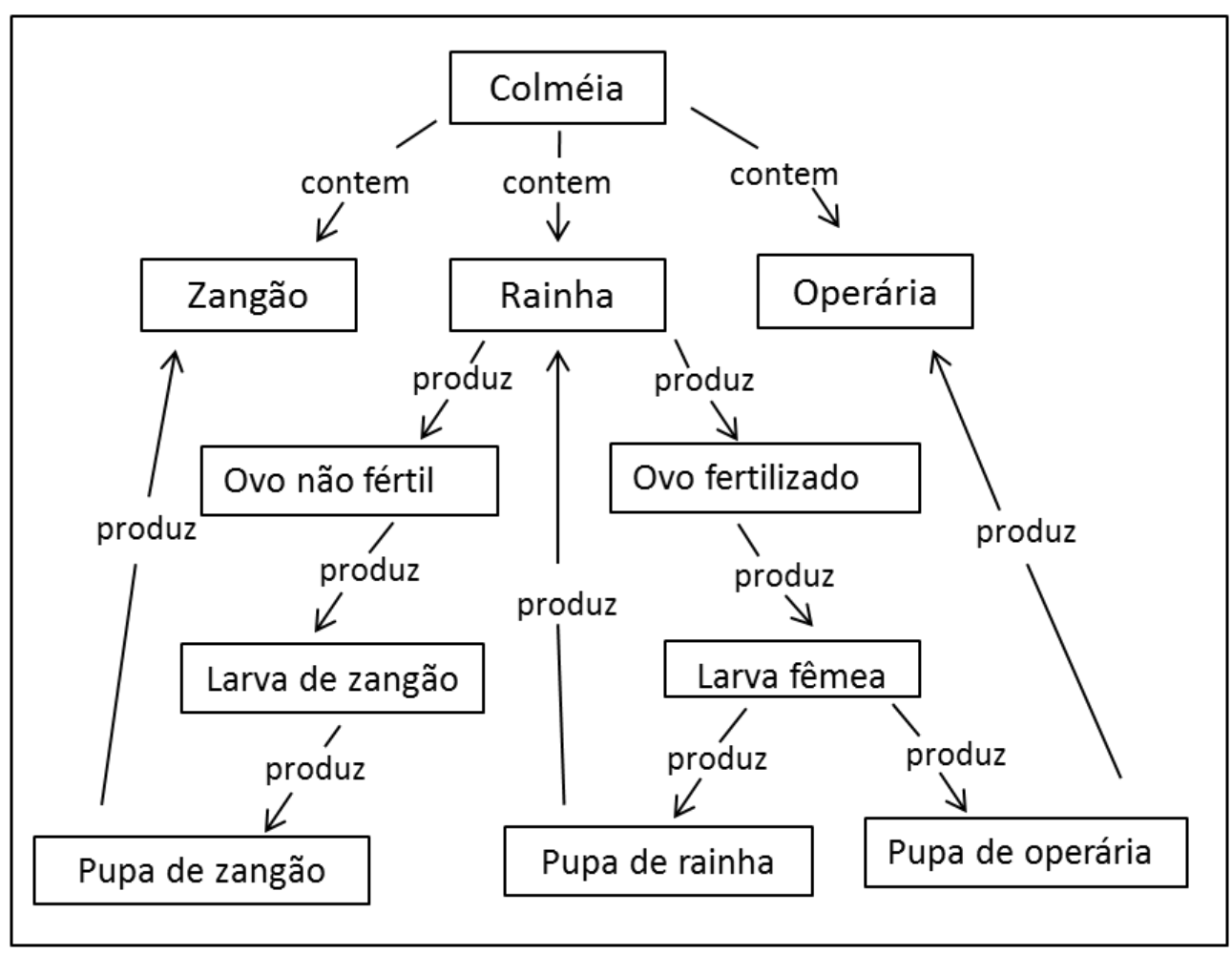

Figura 1 - Exemplo de um Mapa conceitual (Cline, Brewster e Fell, 2010, p. 2283) 
Desta forma, os MC constituem um recurso de aprendizagem significativa, capaz de auxiliar o estudante a integrar novos conceitos a conceitos pré-existentes, estabelecendo estruturas hierárquicas a partir de conceitos mais abrangentes que vão sendo progressivamente relacionados a outros mais específicos. Para o professor, além de servir como uma alternativa para apresentação das aulas, os MC servem também como um meio de avaliação do aprendizado, capaz de evidenciar onde ocorreu aprendizagem satisfatória de conceitos e onde é necessário trabalhar para melhor compreensão.

Este trabalho, realizado com uma turma do $4^{\circ}$ período do curso de Engenharia Química da Universidade Federal do Rio de Janeiro, desenvolveu-se em quatro etapas: na primeira os alunos passaram por um treinamento para utilização da técnica de mapeamento conceitual; na segunda etapa, os MC foram elaborados pelos alunos como parte da primeira avaliação da disciplina de Termodinâmica Clássica do primeiro semestre de 2014; na terceira etapa, entrevistas semi estruturadas foram feitas com 9 alunos com objetivo de identificar suas percepções sobre a técnica do mapeamento conceitual e na quarta etapa os mapas foram analisados através da contagem dos conceitos obrigatórios e complementares e da análise das proposições válidas que foram classificadas como adequadas ou inadequadas do ponto de vista científico.

\section{Mapas conceituais como ferramenta avaliativa}

A avaliação do aprendizado através da utilização de $M C$ é um tema recorrente na área de educação. Apesar de originalmente propostos como um recurso para a avaliação qualitativa que identificasse evidências de uma aprendizagem significativa, os MC também podem ser avaliados em termos quantitativos. Nas palavras de Gowin e Novak (1984, p. 97):

"A pontuação foi, sob muitos aspectos, irrelevante para nós que estávamos procurando por alterações qualitativas na estrutura dos mapas conceituais das crianças. Porém, uma vez que nós vivemos numa sociedade orientada por números, muitos estudantes e professores desejam pontuar os mapas conceituais."

Com intuito de auxiliar o trabalho do professor na avaliação dos MC durante o processo de aprendizagem, Correia, Cicuto e Dazzani (2014) desenvolveram a técnica da Análise de Vizinhança (Aviz). Esta técnica exige que o aluno compulsoriamente utilize alguns conceitos (conceitos obrigatórios) que são selecionados criteriosamente pelo professor. A Aviz utiliza os conceitos obrigatórios para avaliar as proposições elaboradas. Além dos conceitos obrigatórios, os mapas podem apresentar conceitos vizinhos que estão relacionados diretamente com os conceitos obrigatórios e conceitos complementares, os quais não estão relacionados diretamente com os conceitos obrigatórios. A atividade de ensino foi realizada em um colégio particular da região metropolitana de São Paulo, com 39 alunos, divididos em cinco grupos, durante três aulas de 
cinquenta minutos. A atividade foi divida em três etapas. A Etapa 1 da atividade consistiu na leitura de textos sobre o sistema digestório, sistema circulatório e sistema respiratório do corpo humano e obtenção de energia pela célula. A Etapa 2 envolveu a discussão sobre os textos lidos, enquanto a Etapa 3 contemplou a construção de um MC sobre o tema, relacionando os conceitos discutidos na atividade. Os autores propuseram 4 conceitos obrigatórios relacionados ao transporte de nutrientes para a célula: oxigênio, hemácias, células e nutrientes. Foram analisados 36 mapas que continham pelo menos $70 \%$ dos conceitos obrigatórios. Os mapas dos alunos foram comparados a um mapa elaborado pelos autores e, a partir da categorização entre os conceitos obrigatórios e os conceitos vizinhos, procedeu-se a uma contagem do número de proposições que relacionavam esses conceitos. Foram consideradas as categorias "sem sentido" onde as proposições não apresentavam clareza semântica e "erro conceitual" onde são identificadas as proposições inadequadas. Desta forma, a identificação de relações conceituais ingênuas e de erros conceituais é uma forma do professor identificar dificuldades de assimilação dos conceitos e orientar seu trabalho no sentido de um estudo mais preciso.

Uma metodologia distinta foi empregada por Souza e Boruchovitch (2010) para investigar o potencial avaliativo dos MC. Essa pesquisa adotou um estudo de caso com 32 alunas do terceiro ano de um curso de pedagogia na disciplina Fundamentos da Alfabetização. As alunas foram divididas em 16 duplas e tiveram que elaborar seus MC a partir de leituras realizadas durante o curso e não foram fornecidos conceitos obrigatórios. Após a realização dos mapas foi aplicado um questionário exploratório e entrevistas semiestruturadas. O trabalho possibilitou identificar vantagens e limitações (dificuldades) da utilização dos $M C$ com instrumento avaliativo. As principais vantagens citadas pelas alunas foram: (i) identificação das dificuldades de aprendizagem e possibilidade de superação, (ii) favorecimento à reelaboração de conceitos e consequente sedimentação; (iii) integração e ampliação de conhecimentos através da possibilidade de um feedback imediato e (iv) possibilidade de autorregulação e identificação da atividade avaliativa como estratégia de aprendizagem. Por outro lado, as dificuldades enfrentadas pelas alunas referem-se à própria construção dos mapas: identificar, harmonizar e hierarquizar os conceitos estudados e escolher os termos de enlace.

MC foram utilizados para avaliar a evolução do aprendizado de alunos de Engenharia sobre conceitos relacionados ao eletromagnetismo (Moreira, 2013). O autor enfatiza a importância de oportunizar ao aluno a apresentação oral de seu mapa a fim de obter evidências concretas de uma aprendizagem significativa e ressalta também que deve-se ter cuidado ao se comparar MC elaborados pelos alunos com MC construídos por professores ou especialistas admitindo esses como "corretos". Segundo o autor esse seria "um mau uso do mapeamento conceitual por priorizar um enfoque comportamentalista que se afasta das propostas de uma aprendizagem significativa" (Moreira, 2013, p. 35). 
Campos, Ferreira e Gama (2014) analisaram os MC de estudantes de um curso de fonoaudiologia das temáticas das disciplinas de voz (Análise acústica da voz e Distúrbios da voz). Neste trabalho os mapas foram classificados em "satisfatórios", "em desenvolvimento" ou "insatisfatórios" segundo os seguintes critérios: 1) apresentação de acordo com o tema estudado; 2) fácil interpretação e 3) compreensão do tema proposto e 4) presença de ligações entre conceitos diferentes. A avaliação foi realizada pelas autoras por meio do preenchimento de um formulário onde os critérios de avaliação eram descritos detalhadamente. Foram considerados "satisfatórios" os mapas que apresentavam itens de forma completa e correta; "em desenvolvimento" os mapas que apresentavam itens incompletos e "insatisfatórios" os mapas que apresentavam itens incorretos. No entanto, as autoras não deixaram claro quais foram os critérios quantitativos utilizados para se classificar os mapas. Uma vez que, no mesmo mapa, podem ocorrer proposições adequadas e inadequadas (incorretas), qual o número ou a fração de proposições incorretas foram consideradas para se classificar o mapa como "em desenvolvimento" ou "insatisfatório"? Além disso, são apresentados dois exemplos de mapas construídos pelos alunos que relacionam mais de 60 conceitos e com poucas palavras de enlace. Um questionamento imediato com relação a esse trabalho recai sobre a preparação dos alunos para a construção de um mapa conceitual. O fato de um aluno conhecer bem os conteúdos pertinentes a uma determinada temática não o capacita automaticamente para a construção de um bom mapa conceitual. Acredita-se ser necessário um período mínimo de familiarização com a técnica.

Com uma metodologia semelhante àquela adotada por Campos, Ferreira e Gama (2014), Ruiz-Moreno et al. (2007) propõem os MC como instrumentos importantes no monitoramento da aprendizagem, favorecendo uma prática docente baseada na mediação pedagógica. Neste trabalho, desenvolvido em duas disciplinas de um programa de Mestrado em Ciências da Saúde, foram analisados $17 \mathrm{MC}$ produzidos nas disciplinas de Processo de Ensino Aprendizagem e Educação em Saúde. A construção das versões finais dos mapas envolveu diversas etapas de preparação com trabalhos em duplas, apresentação sobre como construir MC, possibilidade de reconstrução e apresentação oral dos mapas. Os mapas deveriam ser construídos com base na bibliografia fornecida pelo professor e não haviam conceitos obrigatórios. Foram considerados os seguintes critérios de análise: 1) Conceitos: quantidade, qualidade e nível de hierarquia conceitual; 2) Inter-relações entre conceitos: linhas de entrecruzamento, número de palavras de enlace e proposições com significado lógico e 3) Estrutura do mapa: sequencial ou em rede, presença de inter-relações não hierárquicas entre segmentos distantes no mapa e criatividade relacionada à estratégia do diagrama. Os autores concluíram que (Ruiz-Moreno et al., 2007, p. 460):

"...os mapas apresentados pelos alunos apresentaram conceitos representativos dos conteúdos abordados em ambas as disciplinas, com uma 
boa hierarquização e com exemplos específicos. Os MC revelaram-se um instrumento importante e inovador para se desenvolver práticas avaliativas comprometidas com a compreensão dos processos de aprendizagem dos alunos".

Cline, Brewster e Fell (2010) criaram um sistema automatizado para avaliação de MC, o Concept Mapping Tool (CMT). Após os estudantes utilizarem o programa para construção do mapa conceitual, eles podem utilizar o sistema de avaliação que compara o mapa construído com o mapa de referência elaborado pelo professor. Desta forma o estudante tem a resposta imediata sobre onde deve melhorar o seu mapa e pode usar o programa interativamente para melhorar sua compreensão dos conceitos estudados. O CMT é constituído pelos seguintes componentes: aplicativo para construção dos mapas, páginas na web para armazenamento dos dados, conjunto de dados que contém informações sobre os mapas dos alunos e do professor e sistema de avaliação. A avaliação feita pelo CMT foi comparada com a avaliação do professor para duas turmas do curso de Entomologia/Biologia da Virginia Polytechnic Institute and State University (Estados Unidos). Na primeira turma da disciplina de Introdução às Abelhas e à Apicultura, foram analisados $15 \mathrm{MC}$. Os resultados mostraram uma boa correlação entre a pontuação atribuída pelo CMT e a pontuação atribuída pelo professor $(r=0,80)$. No entanto, a média da pontuação atribuída pelo CMT foi significativamente menor que a média atribuída pelo professor. Para a segunda turma da disciplina de Biologia dos Insetos, $61 \mathrm{MC}$ foram comparados com relação a avaliação do professor e do CMT. Da mesma forma que no primeiro caso, a correção entre as pontuações foi elevada $(r=0,94)$. Neste caso, porém, as médias não diferiram significativamente.

Com relação à pesquisa em ensino de química, temas como radioatividade (Aquino e De Chiaro, 2013), ligações químicas (Trindade e Hartwig, 2012), equilíbrio químico (Cavalcanti, 2011), conceitos de química orgânica (Freitas, 2007) e proficiência e interpretação de texto para a aprendizagem de transformações químicas (Yano e Amaral, 2011) são alguns exemplos de aplicações dos MC.

Aquino e De Chiaro (2013) investigaram as contribuições dos MC na avaliação do progresso na aprendizagem de conceitos relacionados à radioatividade, comparando, em dois momentos, MC construídos por dois alunos de nível médio, antes e depois de uma intervenção (aula expositiva e dialogada seguida por debate sobre o tema). Os autores concluíram que: "os $M C$ constituem-se um recurso privilegiado de acompanhamento do processo de ensino $e$ aprendizagem dos estudantes por seus educadores" (Aquino e De Chiaro, 2013, p. 158). Esta conclusão foi possível tendo em vista somente o número de conceitos utilizados nos mapas antes e depois da intervenção. De fato, os MC construídos pelos dois alunos após a intervenção apresentaram maior número de conceitos quando comparados aos mapas construídos antes da intervenção. Para o estudante 1, o número de conceitos passou de 7 para 28 e para o estudante 2 , o número de conceitos passou de 10 para 29. No entanto, todos os mapas analisados, antes e 
após a intervenção, não apresentavam qualquer termo de enlace, o que compromete a clareza semântica senão o próprio conceito de proposição. De fato, segundo Correia, Cicuto e Dazzani (2014, p. 134) "a inclusão dos termos de ligação é o principal diferencial dos MC". Sem os termos de enlace o mapa se reduz a um fluxograma comum.

Ligações químicas foi o tema abordado por Trindade e Hartwig (2012) para investigar a estratégia dos $M C$ como ferramenta avaliativa do aprendizado de uma turma de $1^{\circ}$ ano de nível médio. Neste trabalho foi oferecido aos alunos um minicurso de 37 horas sobre ligações químicas, familiarização com MC e revisão de tópicos de estrutura atômica e tabela periódica. Recursos auxiliares como vídeos, textos, modelos e exercícios foram utilizados no minicurso. Foi elaborado um mapa conceitual de referência que foi comparado aos mapas dos alunos considerando 10 categorias de avaliação (conceitos básicos, conceitos novos, ligações entre conceitos, palavras de ligação, exemplos, clareza do mapa, proposições, hierarquização, diferenciação progressiva e reconciliação integrativa). A cada categoria foi atribuído um valor de 0 a 1 e o valor estabelecido como satisfatório para a soma dos valores atribuídos a cada categoria, foi 5,0. As categorias diferenciação progressiva e reconciliação integrativa relacionam-se respectivamente com a hierarquização e com as ligações cruzadas presentes nos mapas e são aspectos essenciais da aprendizagem significativa de Ausubel. Foram elaborados mapas sobre ligações iônicas (38 \% de média satisfatória), ligações covalentes ( 63 \% de média satisfatória) e ligações metálicas (47 \% de média satisfatória). Neste trabalho, no entanto, não fica claro porque os autores em determinado caso fornecem os conceitos básicos e em outro caso não. Para o caso onde os conceitos básicos foram fornecidos, essa categoria não deveria fazer parte da avaliação. Os autores concluíram que "o uso de MC é válido para examinar a organização conceitual dos alunos após um período instrucional..." e sugerem que ..."a metodologia dos MC seja empregada desde os primeiros anos da formação escolar" (Trindade e Hartwig, 2012, p. 90).

Cavalcanti (2011) utilizou os MC para avaliar a compreensão sobre equilíbrio químico dos alunos de um curso de graduação em química em dois momentos: ao ingressarem na universidade e após um ano de curso. Foram também objetivos deste trabalho: descrever e analisar a evolução dos principais conceitos envolvidos e estabelecer uma relação entre as mudanças verificadas nos mapas nos dois momentos diferentes. A utilização dos MC possibilitou identificar as diferenças investigadas. No primeiro momento de avaliação, os alunos definem equilíbrio químico como o estado onde as velocidades das reações se igualam sem fazer qualquer relação com conceitos de termodinâmica, demonstrando, segundo o autor, conhecimentos oriundos de como o conceito é apresentado no ensino médio. Após um ano do curso universitário e serem submetidos a disciplinas relacionadas ao tema equilíbrio químico, a compreensão dos alunos sobre o tema se amplia e aparecem as relações entre energia livre padrão, constante de equilíbrio, quociente de reação e energia livre denotando um aprofundamento no entendimento 
das relações entre espontaneidade de uma reação e da aproximação de um sistema químico do seu estado de equilíbrio.

Conceitos de carboidratos, lipídeos e proteínas foram tratados por Filho (2007) na disciplina de química orgânica em três turmas dos cursos de Agronomia, Medicina Veterinária e Zootecnia da Universidade Federal Rural de Pernambuco. Neste trabalho são apresentados MC construídos pelos alunos durante as atividades programadas em sala de aula ou em laboratório. Os mapas serviram como ponto de partida para discussões e formulação de perguntas que exigiam dos alunos a externalização dos conceitos empregados e das relações entre eles. Por sua versatilidade, os MC foram utilizados como um dos recursos de avaliação em sala de aula.

Yano e Amaral (2011) avaliaram, com auxílio de MC, a aprendizagem de conteúdos de química (transformações químicas) a partir da leitura e interpretação de três textos da Proposta Curricular do Estado de São Paulo (São Paulo, 2011). Para cada texto investigado foram seguidas diversas etapas desde o treinamento na construção de $M C$, a leitura e discussão dos textos e a construção dos mapas. Para o texto 1 - Produção e Uso da Cal - foram selecionados pela professora 25 conceitos; para o texto 2 - Produção do Álcool Combustível - foram selecionados 16 conceitos e para o texto 3 - Produção do Ferro nas Siderúrgicas - foram selecionados 14 conceitos. Com esta seleção de conceitos, a professora construiu um MC de referência ao qual foram comparados os mapas dos alunos segundo os seguintes critérios: número de conceitos; ligações válidas e proposições válidas. Cada critério foi classificado como "muito bom", "satisfatório ou "insatisfatório". Outros critérios como hierarquia, unidade semântica e estrutura global foram analisados em conjunto de acordo com o ponto de vista da professora. As autoras observaram uma "evolução lenta" na análise dos mapas entre os textos 1 e 3 no entanto, não são apresentados resultados quantitativos que justifiquem essa afirmação. Além disso, como os textos são diferentes e foram trabalhados em sequência, a avaliação da aprendizagem e da compreensão por parte dos alunos torna-se subjetiva ou seja, não se pode afirmar com precisão que a "evolução lenta" é resultado de uma melhor compreensão dos textos ou de uma crescente habilidade na construção dos mapas.

Conforme se constata a partir da revisão da literatura, várias metodologias qualitativas e quantitativas são empregadas que abordam os MC como instrumentos de avaliação. Trabalhos em grupo ou individuais, construção de mapas a partir de textos ou a partir de exposições orais, com ou sem conceitos e termos de ligação obrigatórios etc. As avaliações baseiam-se num mapa de referência, critérios de pontuação, contagem das relações diretas e cruzadas entre os conceitos ou em programas automatizados de avaliação. No entanto, independente do instrumento empregado, a falta de rigor metodológico pode acarretar em conclusões inconsistentes, subjetivas ou enviesadas. Para Kinchin (2000, p. 46): 
"Embora numerosos trabalhos reconheçam os MC como uma ferramenta poderosa capaz de facilitar a aprendizagem significativa de conceitos científicos, este recurso ainda não é frequentemente explorado de forma adequada. A explicação para tal evidência pode estar no fato de que os professores ainda não estão certos de como avaliar os mapas de seus alunos".

Por outro lado, no que concerne à preparação dos alunos para utilização do mapeamento conceitual, é essencial um bom treinamento que possibilite a homogeneização da turma no tocante à habilidade para construção dos mapas. Alguns pontos são fundamentais neste treinamento: (i) definição dos termos utilizados (conceitos, termos de ligação, proposições, hierarquia, ligações cruzadas, exemplos, etc...). Neste momento é conveniente a ilustração com um exemplo simples de um mapa conceitual; (ii) definição de uma questão focal ou de um tema que seja de domínio da pessoa que vai elaborar o mapa; (iii) seleção dos diversos conceitos referentes ao assunto em questão procurando ordená-los do mais inclusivo ao mais específico e (iv) elaboração do mapa, cuja construção deve envolver negociação com o grupo, no caso de trabalhos em grupo, ou uma reflexão que procure a melhor organização hierárquica e as ligações cruzadas do mapa e (v) discussão ou apresentação dos mapas construídos.

Outros pontos importantes a serem enfatizados na construção de $M C$ é o favorecimento da autonomia do aluno, o controle sobre seu próprio processo de aprendizagem. Além disso, em função da dinâmica desenvolvida, possibilita a retroalimentação e sedimentação dos conceitos revelando aspectos cognitivos, atitudinais e procedimentais do aluno. São estimuladas as funções cognitivas ligadas a atenção, memória, conceitualização, abstração, comparação, diferenciação, síncrese, análise e síntese.

O empenho e a dedicação durante o processo de construção dos mapas, a preocupação na construção de mapas cada vez mais completos do ponto de vista das proposições e bonitos esteticamente podem também atuar como motivadores para aprender, um aspecto essencial para que se obtenha uma aprendizagem significativa.

\section{Metodologia}

O principal referencial teórico adotado neste trabalho consiste numa adaptação do modelo Avis proposto por Correia, Cicuto e Dazzani (2014). Da mesma forma que no trabalho desses autores, a avaliação dos $\mathrm{MC}$ foi baseada na contagem de conceitos obrigatório e conceitos complementares, porém neste trabalho não foi proposto um $M C$ padrão que servisse de comparação dos mapas em avaliação. 
A metodologia empregada nesta pesquisa se adéqua a um estudo de caso com abordagem quali-quantitativa. Foram investigados 61 alunos de uma turma da disciplina de Termodinâmica Clássica do 4ํ período do curso de Engenharia Química da Universidade Federal do Rio de Janeiro.

A primeira etapa da pesquisa consistiu num treinamento dos alunos para elaboração dos MC. Uma semana antes da avaliação quando se pediria que os alunos elaborassem um mapa, foi proferida uma aula de 120 minutos, cujo tema foi mapeamento conceitual, com os seguintes tópicos: 1 - Breve Histórico; 2 - MC como instrumento de organização e representação do conhecimento; 3 - Apresentações utilizando MC; 4 - Avaliação de MC e 5 - Construção de um mapa conceitual. Ao final da aula os alunos estavam cientes de que uma das questões da avaliação seria a construção de um mapa conceitual envolvendo pelo menos 8 conceitos trabalhados durante as aulas. Foi fornecida bibliografia para aprofundamento no tema constituída de artigos e endereços eletrônicos. Ficou estabelecido na ocasião que as proposições, unidades cognitivas dos MC, deveriam apresentar pelo menos uma palavra de ligação e que as relações entre conceitos sem qualquer palavra de ligação não seriam consideradas na avaliação. Foi ainda disponibilizado um horário para esclarecimento de qualquer dúvida que surgisse.

A segunda etapa da pesquisa consistiu na elaboração dos MC. Na primeira avaliação da disciplina oferecida no primeiro semestre de 2014 foi incluída a seguinte questão sobre conceitos de termodinâmica e mapeamento conceitual: Construa um mapa conceitual com no máximo 12 conceitos relacionando os seguintes conceitos: temperatura, energia, trabalho útil, volume, função de estado, calor, energia interna e trabalho.

Os alunos deveriam elaborar um MC que abordasse pelo menos os 8 conceitos obrigatórios (CO) descritos no enunciado da questão e poderiam utilizar até 4 conceitos complementares (CC) de escolha livre. Os CO foram trabalhados durante as aulas e são descritos propositalmente fora de qualquer ordem hierárquica; coube aos alunos estabelecer a hierarquia entre eles.

Para a avaliação compareceram 61 alunos. Desse total, 3 não fizeram a questão dos mapas e 12 alunos apresentaram mapas que foram excluídos da análise por não conter termos de ligação ou apresentar sentenças como conceitos. Restaram 46 mapas para análise.

Os CO foram numerados de 1 até 8 conforme descrito no Quadro 1 e os CC, adicionados espontaneamente pelos alunos, são descritos no Quadro 2.

178 DOI: Em andamento.

R. Bras. de Ensino de C\&T 
Quadro 1 - Ordenação dos CO

\begin{tabular}{|l|l|}
\hline \multicolumn{2}{|c|}{ Conceitos Obrigatórios - CO } \\
\hline CO1 - energia & CO5 - trabalho \\
\hline CO2 - função de estado & CO6 - temperatura \\
\hline CO3 - energia interna & CO7 - volume \\
\hline CO4 - calor & CO8 - trabalho útil \\
\hline
\end{tabular}

Quadro 2 - CC propostos pelos alunos

\begin{tabular}{|l|l|l|l|}
\hline \multicolumn{4}{|c|}{ Conceitos Complementares - CC } \\
\hline pressão & energia cinética & sistemas abertos & expansão isotérmica \\
\hline trabalho de volume & energia potencial & sistemas fechados & processo isotérmico \\
\hline entalpia & entropia & sistemas isolados & processo isobárico \\
\hline variação de entalpia & variação de entropia & Sistema & processo isocórico \\
\hline $\begin{array}{l}\text { variação de energia } \\
\text { interna }\end{array}$ & variação de volume & $\begin{array}{l}\text { 1a lei da } \\
\text { termodinâmica }\end{array}$ & calor latente \\
\hline termodinâmica & pressão externa & $\begin{array}{l}\text { 2ª lei da } \\
\text { Termodinâmica }\end{array}$ & XXX \\
\hline
\end{tabular}

A terceira etapa da pesquisa consistiu na realização das entrevistas. Foram escolhidos ao acaso e convidados 14 alunos para uma entrevista semiestruturada que se realizou 2 dias após a avaliação. Compareceram 9 alunos para a entrevista a qual teve por objetivo avaliar a familiaridade dos alunos com os MC e suas percepções com relação à metodologia de avaliação adotada.

A quarta etapa da pesquisa foi a análise dos MC elaborados pelos alunos. A contagem e análise das proposições foram feitas da seguinte maneira: por exemplo, para o conceito energia (CO1), foram contadas todas as relações válidas com os demais $\mathrm{CO}$ e com os CC. Para o conceito função de estado (CO2), a contagem foi feita excluindo o conceito energia (CO1) contado anteriormente. Assim a contagem prosseguiu até o conceito trabalho útil (CO8). Cada relação entre os $\mathrm{CO}$ e esses com os $\mathrm{CC}$ foi avaliada como adequada ou inadequada seguindo as normas do que é cientificamente aceito. Por não fazer parte dos objetivos desse trabalho, as relações entre os CC não foram contadas nem avaliadas.

Os principais objetivos desse trabalho foram: (i) investigar a aplicação dos MC como instrumento de avaliação dos conteúdos de termodinâmica clássica; (ii) identificar e avaliar as

DOI: Em andamento. 
proposições construídas pelos alunos entre os $\mathrm{CO}$ e esses com os CC e (iii) investigar a percepção dos alunos com relação a metodologia de avaliação utilizando MC.

\section{Resultados e discussão}

Entre os alunos entrevistados somente um não conhecia os MC. Outro aluno associou MC com fluxogramas e, entre os alunos que declararam conhecer os mapas, as situações nas quais tiveram contato com a metodologia foram em palestras, livros didáticos, no ensino médio e na faculdade.

O Quadro 3 resume as opiniões dos alunos sobre a questão que envolveu a elaboração do mapa conceitual. Adjetivos como fácil, interessante, tranquila, criativa e boa, foram empregados para definir as impressões sobre a questão. Foram feitas alusões também relativas à possibilidade de relação de diferentes conceitos (aluno 9); interligação de conceitos (aluno 4); organização de ideias (aluno 1). Um aluno (5) afirmou que teve dificuldade em organizar as ideias e se limitar a 12 conceitos e outro afirmou que teve dificuldade em encontrar os termos de enlace.

Quadro 3 - Resposta à questão: De modo geral, o que você achou da questão da prova de Termodinâmica Clássica onde teve que utilizar MC?

\begin{tabular}{|c|l|}
\hline Aluno & \multicolumn{1}{|c|}{ Resposta } \\
\hline 1 & "Fácil, gostei, organiza bem as ideias" \\
\hline 2 & "Tranquila, mais fácil que eu esperava" \\
\hline 3 & "Interessante, demonstra que a pessoa compreendeu os conceitos" \\
\hline 4 & "Forma criativa de avaliar interliga conceitos, não é só decorar fórmulas" \\
\hline 5 & "Tive dificuldade em organizar as ideias e me limitar a 12 conceitos" \\
\hline 6 & "Interessante, melhor do que cobrar dedução de fórmulas" \\
\hline 7 & "Achei a questão boa, outros professores deveriam cobrar também" \\
\hline 8 & $\begin{array}{l}\text { "É uma boa maneira de avaliar se o aluno compreendeu as relações entre os } \\
\text { conceitos" }\end{array}$ \\
\hline 9 & "Mostrou que se podem relacionar diferentes conceitos" \\
\hline
\end{tabular}

Quando indagados se outro professor havia utilizado os MC e em que circunstância (aula ou avaliação) os mapas teriam sido utilizados, dos 9 entrevistados, 5 responderam que nenhum outro professor havia utilizado, 2 responderam que os mapas foram utilizados somente em aula e 2 responderam que os mapas foram utilizados em aula e em prova.

De modo geral, foi positiva a receptividade dos alunos com relação à questão envolvendo a construção do mapa conceitual. As entrevistas revelaram dificuldades que deverão ser trabalhadas principalmente durante o período de capacitação para elaboração dos MC. As entrevistas evidenciaram também que esta ferramenta auxilia aos alunos na compreensão dos 
conceitos e da teoria como um todo, não se limitando, como dito pelos próprios alunos, a uma aprendizagem mecânica baseada na memorização e aplicação de fórmulas.

O Quadro 4 apresenta, para cada CO, o número total de proposições, o número e a porcentagem de proposições adequadas e o número de mapas e a porcentagem onde não havia proposições relacionadas ao $\mathrm{CO}$. Cabe ressaltar que as proposições que não apresentavam nenhum termo de enlace foram contadas como ausentes.

Quadro 4 - Total de proposições por conceito obrigatório

\begin{tabular}{|c|c|c|c|}
\hline Conceito & $\begin{array}{c}\text { Total de } \\
\text { Proposições }\end{array}$ & $\begin{array}{c}\text { Proposições } \\
\text { Adequadas (\%) }\end{array}$ & $\begin{array}{c}\text { Ausência de } \\
\text { Proposições (\%) }\end{array}$ \\
\hline CO1 & 69 & $62(89,9 \%)$ & $5(10,9 \%)$ \\
\hline CO2 & 59 & $54(91,5 \%)$ & $7(15,2 \%)$ \\
\hline CO3 & 104 & $79(76,0 \%)$ & $4(8,7 \%)$ \\
\hline CO4 & 77 & $58(75,3 \%)$ & $2(4,3 \%)$ \\
\hline CO5 & 96 & $80(83,3 \%)$ & $3(6,5 \%)$ \\
\hline CO6 & 54 & $44(81,5 \%)$ & $1(2,2 \%)$ \\
\hline CO7 & 48 & $42(87,5 \%)$ & $2(4,3 \%)$ \\
\hline CO8 & 50 & $47(94,0 \%)$ & $4(8,7 \%)$ \\
\hline
\end{tabular}

*Em relação aos 46 mapas analisados

De uma maneira geral, foi baixa a ausência dos CO - fato que em si já revela a preocupação dos alunos em construir um mapa "completo" - variando, em números percentuais relativos ao total de mapas avaliados, de 2,2 \% para o CO6 (temperatura) até 15,2 \% para o CO2 (função de estado). Embora todos os conceitos tenham sido tratados durante as aulas, essa diferença obtida pode ser atribuída aos conhecimentos prévios trazidos pelos alunos. Certamente, no senso comum, temperatura é um conceito mais utilizado que função de estado. Em números absolutos, o $\mathrm{CO} 3$ (energia interna) foi o conceito mais frequente. Na interpretação da maioria dos alunos a energia interna foi o conceito mais inclusivo, de onde partiram ou chegaram setas formando o maior número de proposições. De fato, o estudo da 1aㅡ Lei da Termodinâmica, na maioria dos livros didáticos e na forma como foi enfatizado nas aulas, parte da definição deste conceito como uma propriedade do sistema cuja variação relaciona-se diretamente ao calor e ao trabalho. Volume ( $\mathrm{CO} 7$ ) e trabalho útil ( $\mathrm{CO} 8$ ) foram os conceitos menos frequentes, interpretados como menos inclusivos pelos alunos. Com relação à porcentagem de proposições adequadas, a maioria dos CO apresentou índices superiores a $80 \%$ indicando que os alunos demonstraram compreender bem a construção do mapa e as relações entre os CO. Os conceitos energia interna (CO3), calor ( $\mathrm{CO} 4)$ e temperatura (CO6) apresentaram maiores proporções de proposições inadequadas. Esses resultados serão tratados na análise das relações entre os CO. 
O Quadro 5 apresenta todas as proposições válidas (adequadas e inadequadas) elaboradas pelos alunos, relacionando os CO e esses com os CC. Para o total de 353 proposição válidas, 298 $(84,4 \%)$ foram analisadas como adequadas mostrando que o grupo de alunos investigado teve uma boa compreensão das relações entre os conceitos na construção dos MC.

Quadro 5 - Proposições entre os $\mathrm{CO}$ e os CC

\begin{tabular}{|c|c|c|c|c|c|}
\hline Conceitos & $\begin{array}{l}\text { Proposições } \\
\text { Adequadas }\end{array}$ & $\begin{array}{l}\text { Proposições } \\
\text { Inadequadas }\end{array}$ & Conceitos & $\begin{array}{l}\text { Proposições } \\
\text { Adequadas }\end{array}$ & $\begin{array}{l}\text { Proposições } \\
\text { Inadequadas }\end{array}$ \\
\hline $\mathrm{CO} 1-\mathrm{CO} 2$ & 3 & 1 & $\mathrm{CO3}-\mathrm{CO} 7$ & 2 & 1 \\
\hline $\mathrm{CO} 1-\mathrm{CO}$ & 18 & 1 & $\mathrm{CO3}-\mathrm{CO} 8$ & 1 & 0 \\
\hline C01 - C04 & 15 & 1 & $\mathrm{CO}-\mathrm{CC}$ & 14 & 2 \\
\hline $\mathrm{CO} 1-\mathrm{C} 05$ & 6 & 0 & $\mathrm{CO} 4-\mathrm{CO} 5$ & 0 & 0 \\
\hline C01 - C06 & 5 & 1 & C04 - C06 & 12 & 4 \\
\hline $\mathrm{CO1}-\mathrm{CO} 7$ & 1 & 1 & $\mathrm{CO} 4-\mathrm{CO} 7$ & 0 & 0 \\
\hline $\mathrm{CO1}-\mathrm{C} 08$ & 1 & 0 & $\mathrm{CO4}-\mathrm{CO} 8$ & 1 & 0 \\
\hline $\mathrm{CO1}-\mathrm{CC}$ & 13 & 2 & $\mathrm{CO} 4-\mathrm{CC}$ & 17 & 1 \\
\hline $\mathrm{CO} 2-\mathrm{CO}$ & 24 & 0 & C05 - C06 & 0 & 0 \\
\hline $\mathrm{CO} 2-\mathrm{CO} 4$ & 3 & 1 & $\mathrm{CO5}-\mathrm{CO}$ & 10 & 0 \\
\hline $\mathrm{CO}-\mathrm{CO} 5$ & 4 & 0 & $\mathrm{C05}-\mathrm{C0} 8$ & 27 & 1 \\
\hline $\mathrm{CO} 2-\mathrm{CO}$ & 3 & 1 & $\mathrm{CO} 5-\mathrm{CC}$ & 23 & 4 \\
\hline C02 - C07 & 2 & 1 & $\mathrm{CO6}-\mathrm{CO} 7$ & 1 & 0 \\
\hline $\mathrm{CO} 2-\mathrm{CO} 8$ & 1 & 0 & $\mathrm{C06}-\mathrm{C08}$ & 0 & 0 \\
\hline $\mathrm{CO} 2-\mathrm{CC}$ & 14 & 1 & C06 - CC & 13 & 3 \\
\hline $\mathrm{CO}-\mathrm{CO} 4$ & 10 & 12 & $\mathrm{C} 07-\mathrm{C} 08$ & 8 & 1 \\
\hline $\mathrm{CO3}-\mathrm{CO} 5$ & 10 & 11 & $\mathrm{CO} 7-\mathrm{CC}$ & 18 & 2 \\
\hline C03 - C06 & 10 & 1 & $\mathrm{CO} 8-\mathrm{CC}$ & 8 & 1 \\
\hline
\end{tabular}

Duas relações, $\mathrm{CO} 3$ - $\mathrm{CO} 4$ e $\mathrm{CO} 3$ - $\mathrm{CO} 5$, destacam-se quando o número de relações adequadas é comparado com o número de relações inadequadas. De fato, as proposições envolvendo energia interna ( $\mathrm{CO} 3)$ e calor $(\mathrm{CO} 4)$ e as proposições envolvendo energia interna (CO3) e trabalho (CO5) apresentaram maior número de proposições inadequadas do que adequadas. Este é um resultado importante na medida em que permite identificar concepções incorretas elaboradas pelos alunos. De fato, a maioria das relações inadequadas relacionava a energia interna - e não a variação da energia interna - com calor e trabalho. A energia interna, enquanto propriedade dos sistemas, não pode ser relacionada com calor e trabalho que não são propriedades dos sistemas. Esta concepção errônea foi a principal responsável pela menor proporção de proposições adequadas de $\mathrm{CO} 3$ e $\mathrm{CO} 4$ apresentadas no Quadro 4. De fato, a energia interna, conceito mais utilizado pelos alunos, foi frequentemente relacionado com calor e 
trabalho como uma alusão ao primeiro princípio da termodinâmica sem a reflexão de que o termo correto seria variação de energia interna.

Por outro lado, o calor, conceito muito utilizado no senso comum como integrante dos sistemas - é comum as expressões "hoje está calor" ou "estou com calor"... - também apresentou, comparativamente, menor percentual de proposições adequadas. De acordo com Pozo e Crespo (2009, p. 201), "os alunos, assim como ocorria nas primeiras teorias históricas, atribuem propriedades materiais ao calor e consideram que é um tipo de substância que pode passar de um corpo para outro".

Embora numa proporção menor, as proposições construídas entre $\mathrm{CO} 4$ e $\mathrm{CO} 6$ (calor e temperatura) também apresentaram um número expressivo de relações inadequadas. Das 16 relações, 4 (25,0 \%) foram inadequadas. Os alunos que mantém a conviç̧ão na essência material do calor tendem a considerar calor e temperatura como sinônimos, diretamente relacionados com as características dos sistemas. Assim, corpos diferentes em equilíbrio térmico, apresentariam temperaturas diferentes dependendo do tamanho ou da sua natureza. Esta é uma concepção errônea bastante comum no cotidiano (Pozo e Crespo, 2009).

As relações elaboradas entre trabalho (CO5) e trabalho útil (CO8) foram as mais frequentes nos mapas analisados. Foram classificadas como adequadas 27 das 28 relações válidas entre esses conceitos. Esses conceitos, não sendo tão presentes no senso comum, parecem não ter sofrido influencia de concepções prévias errôneas.

Por outro lado, as relações $\mathrm{CO} 4$ - CO5, $\mathrm{CO} 4$ - CO7, $\mathrm{CO} 5$ - $\mathrm{CO} 6$ e $\mathrm{CO} 6$ - $\mathrm{CO} 8$ não apareceram. Calor e trabalho, embora relacionados frequentemente com a energia interna ou com a variação da energia interna, não aparecem relacionados entre si. Os alunos também não identificaram relações entre calor e volume, entre trabalho e temperatura e entre temperatura e trabalho útil.

A Figura 2 apresenta 2 MC elaborados pelos alunos que apresentaram proposições inadequadas. Na Figura 2a existe a associação direta entre a energia interna - e não a variação da energia interna - com o calor. Além disso, a proposição "CALOR - quando em fluxo chama-se TEMPERATURA" demonstra uma concepção em desacordo com os conceitos científicos de calor e temperatura. Na Figura $2 \mathrm{~b}$ a energia interna está corretamente associada com o somatório das energias que compõem o sistema, porém as proposições "ENERGIA INTERNA - deriva do - CALOR" e "ENERGIA INTERNA - deriva do - TRABALHO" foram classificadas como inadequadas do ponto de vista científico. Esses mapas evidenciaram a necessidade de um trabalho adicional do professor no sentido da correção dessas concepções incorretas por parte dos estudantes. 
Figura 2 - Exemplos de MC elaborados pelos alunos.

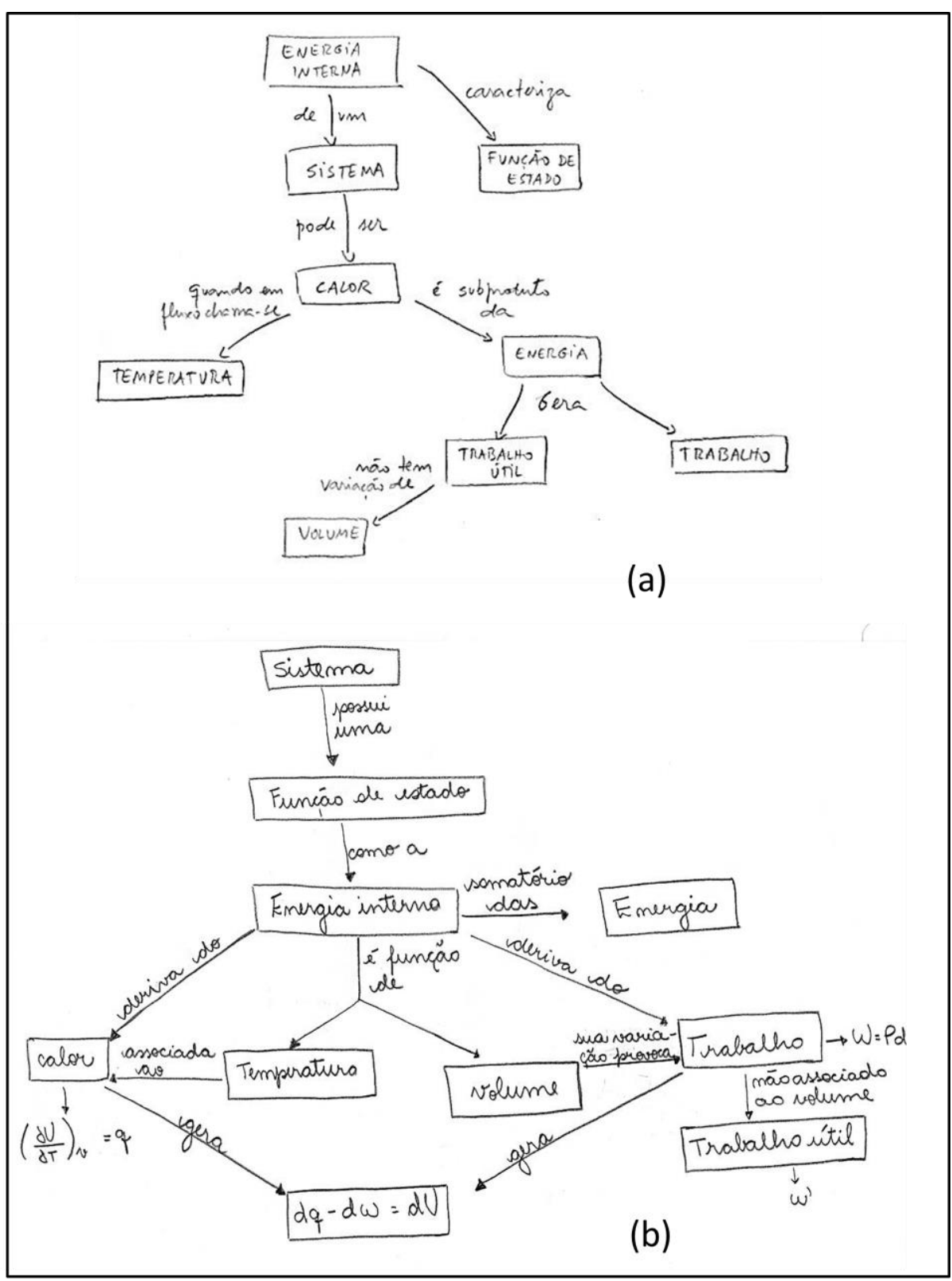

\section{Conclusões e considerações finais}

Neste trabalho a utilização de MC para avaliação de conceitos de termodinâmica clássica revelou-se uma ferramenta capaz de evidenciar concepções adequadas e inadequadas dos estudantes. Entre todas as proposições válidas - proposições que ligavam dois conceitos por um termo de enlace - $84,4 \%$ foram analisadas como adequadas isto é, em conformidade com o que é cientificamente aceito. Este resultado revelou uma boa compreensão por parte dos estudantes das relações entre os $\mathrm{CO}$ e esses com os $\mathrm{CC}$. 
A proporção de ausência dos CO foi baixa, variando de 2,2 \% para proposições que envolvessem o conceito temperatura até $15,2 \%$ para proposições que envolvessem o conceito de função de estado. Este resultado revelou que os alunos efetivamente procuraram construir mapas completos e com proposições válidas.

A avaliação das proposições elaboradas pelos alunos revelou que o CO mais frequente foi energia interna e o menos frequente foi volume. Apesar de mais frequente, o conceito energia interna foi um dos que apresentaram menor proporção de proposições adequadas. Um percentual importante dos mapas analisados relacionou diretamente energia interna com calor $\mathrm{e}$ trabalho. Este resultado foi importante para revelar uma concepção errônea que associa calor e trabalho com propriedades dos sistemas. Ficou evidente ser necessário trabalhar mais essas relações para uma melhor compreensão dos alunos. Essas relações, por envolverem conceitos bastante difundidos no senso comum, foram atribuídas às concepções prévias errôneas - do ponto de vista científico - herdadas pelos alunos do conhecimento cotidiano.

As percepções dos alunos com relação à utilização dos $M C$ investigadas nas entrevistas semiestruturadas revelaram uma boa aceitação dessa técnica. Os alunos que afirmaram ter tido alguma dificuldade para elaborar o mapa apresentaram como justificativa o fato de não encontrarem os termos de enlace ou a limitação a 12 conceitos. Essas dificuldades relacionam-se principalmente aos conteúdos da disciplina e não à construção dos mapas. Assim, o procedimento de treinamento para elaboração dos $\mathrm{MC}$ foi adequado para que os alunos aprendessem as noções básicas de como trabalhar com a técnica.

\section{Referências}

AQUINO, K. A. e DE CHIARO, S. Uso de MC: percepções sobre a construção de conhecimentos de estudantes do ensino médio a respeito do tema radioatividade. Ciência e Cognição, v. 18, n. 2, p. 158-171, 2013.

CAVALCANTI, R. R. Desenvolvimento e aplicação de um método de análise de MC com objetivo de acompanhar mudanças na compreensão de grupos de alunos sobre o tema equilíbrio químico, 2011, 92 f. Dissertação (MESTRADO) - Programa de Pós-Graduação Interunidades em Ensino de Ciências Modalidade Química - Universidade de São Paulo, São Paulo, 2011.

CAMPOS, N. F., FERREIRA, J. M. e GAMA, A. C. Mapa conceitual: ferramenta didática no curso de fonoaudiologia. Distúrbios de Comunicação, v. 26, n. 1, p. 110-117, 2014.

CLINE, B. E., BREWSTER, C. C. e FELL, R. D. A rule-based system for automatically evaluation student concept maps. Expert systems with applications, v. 37, p. 2282-2291, 2010.

CORREIA, P. R., CICUTO, C. A. e DAZZANI, B. Análise de vizinhança de mapas conceituais a partir do uso de múltiplos conceitos obrigatórios. Ciência e Educação, v. 20, n. 1, p. 133-146, 2014.

R. B. E. C. T., vol 8, núm. 4, set-dez.2015 ISSN - 1982-873X

DOI: Em andamento. 
FREITAS, J. R. Mapas conceituais: estratégia pedagógica para construção de conceitos na disciplina de química orgânica, Ciência \& Cognição, v. 12, p. 86-95, 2007.

GOWIN, B. e NOVAK, D. Aprender a aprender. Lisboa: Plátano Editora, 1984.

KINCHIN, I. M. Using concept maps to reveal understanding: a two-tier analysis, Scholl Science Review, v. 81, p. 41-46, 2000.

MOREIRA, M. A. Aprendizagem significativa em mapas conceituais, Textos de Apoio ao Professor de Física, v. 24, n. 6, p. 1-53, 2013.

NOVAK, J. D. e CAÑAS, A. J. The Theory Underlying Concept Maps and How to Construct Them, Technical Report IHMC CmapTools, Florida Institute for Human and Machine Cognition, Disponível em: <http://cmap.ihmc.us/Publications/ResearchPapers/TheoryUnderlyingConceptMaps.pdf>, Acesso em: 25 mar. 2014.

POZO, I. P. e CRESPO, M. A. A aprendizagem e o ensino de ciências. Porto Alegre: Artmed, 2009.

RUIZ-MORENO, L., SONZOGNO, M. C., BATISTA, S. H. e BATISTA, N. A. Mapa conceitual: ensaiando critérios de análise, Ciência \& Educação, v. 13, n. 3, p. 453-463, 2007.

SÃO PAULO (Estado) Secretaria da Educação, Currículo do Estado de São Paulo: Ciências da Natureza e suas tecnologias, Secretaria da Educação, São Paulo: SE, 2011, 152 p.

SOUZA, N. A. e BORUCHOVITCH, E. Mapa conceitual: seu potencial como instrumento avaliativo, Pró-Posições, v. 21, n. 3, p. 173-192, 2010.

TRINDADE, J. O. e HARTWIG, D. R. Uso combinado de mapas conceituais e estratégias diversificadas de ensino: uma análise inicial das ligações químicas, Química Nova na Escola, v. 34, n. 2, p. 83-91, 2012.

YANO, E. O. e AMARAL, C. L. Mapas conceituais como ferramenta facilitadora na compreensão e interpretação de textos de química, Experiências em Ensino de Ciências, v. 6, n. 3, p. 76-86, 2011.

Guilherme Cordeiro da Graça de Oliveira - Doutor em Engenharia Metalúrgica e de Materiais COPPE/UFRJ. Docente do Programa de Pós-Graduação em Ensino de Química - Instituto de Química da UFRJ.cordeiro@iq.ufri.br

Paula Macedo Lessa dos Santos - Doutora em Química de Produtos Naturais pela UFRJ. Docente do Programa de Pós-Graduação em Ensino de Química - Instituto de Química da UFRJ. paulalessa@iq.ufri 
Rodrigo dos Santos Almeida - Mestre em Ciências e Tecnologias Nucleares - Instituto de Engenharia Nuclear. Docente e Coordenador do Curso Técnico em Química do IFRJ. rodrigo.almeida@ifri.edu.br 